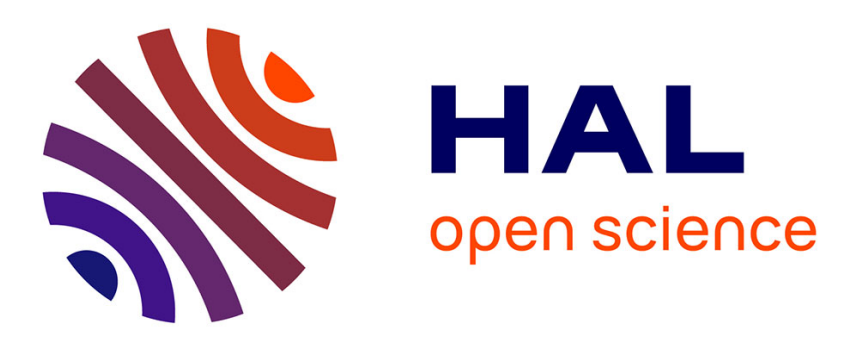

\title{
Symmetry-Induced Deformation and Reconstructive Phase Transformation in Metal-Oxide Interface: The Fe (001) example
}

\author{
L. Lahoche, V. Lorman, J. Roelandt, S. Rochal
}

\section{- To cite this version:}

L. Lahoche, V. Lorman, J. Roelandt, S. Rochal. Symmetry-Induced Deformation and Reconstructive Phase Transformation in Metal-Oxide Interface: The Fe (001) example. Journal de Physique IV Proceedings, 1996, 06 (C1), pp.C1-129-C1-138. 10.1051/jp4:1996113 . jpa-00254145

\section{HAL Id: jpa-00254145 https://hal.science/jpa-00254145}

Submitted on 1 Jan 1996

HAL is a multi-disciplinary open access archive for the deposit and dissemination of scientific research documents, whether they are published or not. The documents may come from teaching and research institutions in France or abroad, or from public or private research centers.
L'archive ouverte pluridisciplinaire $\mathbf{H A L}$, est destinée au dépôt et à la diffusion de documents scientifiques de niveau recherche, publiés ou non, émanant des établissements d'enseignement et de recherche français ou étrangers, des laboratoires publics ou privés. 


\title{
Symmetry-Induced Deformation and Reconstructive Phase Transformation in Metal-Oxide Interface: The Fe (001) example
}

\author{
L. Lahoche*,**, V. Lorman*, J.M. Roelandt** and S.B. Rochal*** \\ * Laboratoire de Physique de la Matière Condensée, Université de Picardie Jules Verne, Amiens, France \\ ** Division Modèles Numériques en Mécanique, Laboratoire de Génie Mécanique pour les Matériaux \\ et les Structures, Université de Technologie de Compiègne, France \\ *** Institute of Physics, Rostov State University, Rostov-on-Don, Russia
}

\begin{abstract}
A model is proposed for the structural transformation and corresponding induced deformation in physical three-dimensional interface of the metal-oxide system. The thermodynamical and elastic state of the system is described by the Landau-Ginzbourg free energy. Calculated theoretical phase diagram shows several different types of isothermal growth processes. The model is applied to the case of the oxidation of the (001) Fe surface.
\end{abstract}

\section{INTRODUCTION}

Formation of the oxide scale on high temperature alloys is a factor favouring protection of the metal. Protective properties depend strongly on the adherence of the oxide scale, its density and compact structure [1]. It is well known that the process of oxidation can induce deformation of unstressed metal [24] or alter the deformation of material submitted to stresses during its use [5]. The main cause of these effects is thought to be the stresses that may exist in the oxide, in the substrate and at the metal-oxide interface. Many review articles [6-11] provided information on the origins and development of the residual stresses in the metal-oxide systems: variation of the volume between the metal and its oxide characterized by the Pilling-Bedworth Ratio (PBR), epitaxic phenomena, variation of thermal expansion due to the difference of the metal and oxide expansion coefficients, changes in the composition, real geometry of the sample etc.

Although possible stress sources have been studied extensively, the influence of the stress generation during isothermal oxidation (growth stresses) and during cooling (thermal stresses) is not clear yet. In the last few years several new stress investigation techniques have been developed, which allow to follow the stress during heating-cooling sequences. The most powerful of them is the X-ray diffraction method [1218]. However, this method is not direct, and is based on the diffraction measurement of the film lattice parameter ( or its spatial distribution), e.g. on a strain measurement. The internal stresses are then recalculated using $X$-ray crystallographic elastic constants.

One of the advantages of this method is the possibility to detect phase transformation, which may take place in the scale or in one of its parts during growth process; it can be done simultaneously with the strain measurements both in the case of displacive or order-disorder type phase transition. The phase transitions (especially the first-order phase transitions) in crystals or ceramics lead often to their fracture. The main fracture origin is the spontaneous deformation induced by the order parameter of corresponding phase transition (so-called symmetry-induced deformation), which give rise to stress concentration at the inhomogeneities[19,20]. Oxide scale, submitted, on the one hand, to the anisotropic internal field due to the interaction with the metallic substrate, and on the other hand to the oxygen-oxygen interaction resulting mainly in the isotropic elastic field, can undergo phase transition. Such kind of phenomena should be evidently temperature- and thickness-dependent. Moreover, several experimental indications speaking in favour of the possible phase transition in the oxide scale during its growth process have been already reported (see f.e., $[14,17]$ ), namely, non-monotonous dependence or anomaly of the strain, measured by the $\mathrm{X}$-ray diffraction, as the function of the thickness of the oxide layer, spontaneous anisotropy of the elastic stiffness tensor etc. 
Both, resulting stresses and possibility of phase transition in the oxide scale are closely related to corresponding epitaxial metal/oxide relationships. Actually, the lattice "mismatch "during oxidation can give rise to stresses within the scale [21]. Epitaxially induced stresses are usually the only to be considered significant for scale thicknesses of less then $0.02 \mu \mathrm{m}$ [22]; large stresses in the thick scales formed on an austenitic stainless steel have been also attributed to this source $[23,24]$. On the other hand, metal-metal and metal-oxygen interactions defined by epitaxial relationships can induce phase transition of the orderdisorder type. Furthermore, epitaxial relationships should determine the crystallographic structure of the ordered intermediate phase which can be evidently considered as three-dimensional (3D) metal-oxide interface. Coupling of the order parameter of the phase transition assisted by cation diffusion process with elastic degrees of freedom leads to the "symmetry induced " deformation of the interface, which contributes in turn to the scale stresses.

The aim of this paper is to present a model of the structural phase transformation and corresponding symmetry-induced deformation in physical three-dimensional interface of the (metal substrate)-(oxide scale) system with dominant cation diffusion process. We show that with the help of epitaxial relationships (orientational relationship of the metal and oxide lattices together with ratio of the lattice parameters) one can choose crystallographic structure of the 3D-interface, estimate maximal value of the symmetry-induced deformation and related "effective" PBR value. Crystallographic structure of the interface permits to determine the wave vector of the ordering of metallic ions in the oxygen matrix and corresponding order parameter, supposing that in the thin oxide film this ordering is imposed by the thick metallic substrate. The difference of the oxygen-oxygen interatomic distances in the atomic layer, which satisfies epitaxial relationship and those in free oxide is taken to be the source of isotropic elastic constrains. This stress field applied to the 3D-interface is an internal field of the scale and is a function of the scale thickness. Thermodynamic and elastic state of the system is described by the free energy of the Landau-Ginzburg type [25], corresponding calculated theoretical phase diagram is related to the experimental temperaturethickness diagram and can show different types of growth processes for different temperatures of oxidation.

The main principles of the model are illustrated on the example of the oxidation of the (001) Fe surface. It is shown that the structure of the 3D-interface in this case is four-layered tetragonal one, with the space group $\mathrm{D}_{4 \mathrm{~h}}^{1}(\mathrm{P} 4 / \mathrm{mmm})$. Corresponding six-component order parameter expresses the difference of the occupation probabilities of the oxygen lattice interstices by $\mathrm{Fe}$ ions and is associated with the wave vector $\vec{k}=\frac{1}{4}\left(\vec{b}_{1}+\vec{b}_{2}\right)$, where $\vec{b}_{1}$ and $\vec{b}_{2}$ are the basic vectors in the reciprocal space of the f.c.c. lattice of oxygen. Maximal symmetry-induced deformation is shown to be $5,8 \%$ and "effective" PBR value varies from 1.12 to 1.19 according to the type of the growth process defined by the phase diagram, although classical PBR value for the $\mathrm{Fe} / \mathrm{FeO}$ system is $1.68[8,26]$. Calculated temperature-internal field phase diagram shows variety of growth regimes : isotropic growth without phase transition, second order phase transition without structural incompatibility of the substrate and the interface, first order phase transition resulting in the loss of elastic continuity and grain formation.

The paper is organized as follows. As a preliminary section, a unified phenomenological description of the 3D metal-oxide interface is given (Sec. 2.1), based on the group theory and atomistic analysis. The method is then applied to the case of the oxidation of the (001) Fe substrate (Sec. 2.2). The obtained results on the structural characteristics and different growth regimes are briefly discussed (Sec.3) in relation with following applications.

\section{STRUCTURAL CHARACTERISTICS AND PHASE TRANSITION IN THE METAL-OXIDE INTERFACE}

\subsection{General}

We start with the analysis of possible crystallographic structure of the metal-oxide interface. Usual methods used for that purpose are derived from the theory of grain boundaries in crystals (see f.e. [2729]). The basis of the concept is the Coincidence Site Lattice (CSL) [30-33], and the crystallographic theory of O-lattice $[34,35]$. This theory is a mathematical method for calculating the crystallographic structure of interfaces between two arbitrary crystals in arbitrary relative orientation. However, physically the problem is equivalent to searching for the interface structure of minimum energy which, of course 
cannot be found by geometrical means only. It should be pointed out aiso, that geometrical considerations have neglected the effect of temperature, pressure etc. on the boundary structure. There is substantial evidence in the literature $[36,37]$ to suggest structural phase transformations in grain boundaries and interfaces $[38,39]$. This hypothesis is confirmed by measurements of the orientation relationships corresponding to boundaries of low energy as a function of temperature [40] and pressure [41].

In the present study we propose to take into account geometrical relations between the lattices of the substrate and of the oxide in a certain extend. Namely, the structure of the 3D-interface must represent common substructure of the substrate and of the oxide, but up to all possible symmetry-nonbreaking deformations. This choice implies that the symmetry space group $G_{I}$ of the $3 D$-interface must be common subgroup of the space groups of the substrate $G_{m}$ and of the oxide $G_{o x}$, which satisfies corresponding orientation relationship and lattice parameters ratio. This relation is also valid only up to all symmetrynonbreaking deformations. This restriction, which is usual in the theory of structural phase transitions [see, f.e., 42-45], allows the lattices to be not in rigid coincidence (or near coincidence), but to be only correlated from the group theory point of view. In this approach, the equilibrium structure of the 3Dinterface depends, evidently, on external parameters (temperature, pressure, scale thickness, composition etc.), and should be determined by the minimization of the free energy $\bar{F}$ of the interface, invariant with respect to corresponding symmetry space group.

In general case it is very difficult to find invariant free energy, which can describe simultaneously metal substrate, 3D-interface and equilibrium oxide, because the space groups of the substrate and of the oxide have usually no group-subgroup relationship. In the theory of structural phase transitions this difficulty is usually overcome with the help of the parent phase concept[42-45]. Parent phase is the degenerate structure (in many cases only hypothetical or "latent" one), from which both real structures can be obtained by way of phase transition, but with two different order parameters. In reality, it is a method to find the common origin of all the degrees of freedom of thermodynamic system, involved into the phase transformations. Unfortunately, for f.c.c. and b.c.c. structures, which is the case of many metals and their oxides, corresponding parent phase cannot be crystallographic structure and the transition is reconstructive. Recently, relative progress has been achieved in the description of the reconstructive phase transitions with the help of the notion of nonlinear order parameter [46-48].

However, in the case of the metal-oxide interface with dominant cation diffusion process the significant simplification can be made. Due to the fact that the oxide lattice in this case does not practically penetrate into the substrate lattice one can choose as the parent phase for the oxide and for the 3D-interface hypothetical oxygen cubic lattice, which satisfies exactly epitaxial relationship between the oxygen sublattice of the oxide and the lattice of the metal substrate. Then, the structure of the 3D-interface is obtained by the ordering of the metallic ions in the interstices of the oxygen matrix, assisted by the cation diffusion mode. On the other hand, the structure of the interface must satisfy orientational relationship and lattice parameters ratio with equilibrium oxide lattice. Of course, this approach is applicable only to the thin films, in which the correlation length of the anisotropic diffusion mode $\zeta>\mathrm{d}$, where $\mathrm{d}$ is the scale thickness. Thus, the orientation relationships determine the wave vector of the reciprocal space of the oxygen lattice, which is associated with the order parameter. Order parameter expresses the difference of probabilities for the interstices in different layers to be occupied by metallic ions. The components of the order parameter span an irreducible representation of the space group of the oxygen lattice. Corresponding thermodynamical free energy must be invariant with respect to this representation.

In the same manner the concept of the parent phase have to be applied to the elastic degrees of freedom of the metal-oxide interface, in order to find the common origin of all the thermo-mechanical degrees of freedom. It implies the choice of the oxygen lattice as the stress-free non-deformed state. Formally, in the present model, the components of the strain tensor $\varepsilon_{\mathrm{ij}}$, induced by the phase transition through the oxidation process are measured with respect to this state. Analogously, internal stress $\sigma_{\mathrm{ij}}$ existing in the interface is applied to this state. The internal stress $\sigma_{\mathrm{ij}}$ in the model is due to the oxygen-oxygen interaction, which tends to put oxygen atoms at the distance equal to that in the equilibrium free oxide. This internal stress is applied to the oxygen lattice of the parent phase in such a way, that it is equal to zero in the first epitaxial layer of the oxygen, it grows with the thickness of the scale and it reaches some constant value when the distance between oxygen attends equilibrium. 
Symmetry-induced deformation appears in the scale due to the thermo-mechanical coupling of the order parameter with elastic degrees of freedom. The terms in the free energy, which are responsible of this coupling are presented by the mixed invariants, composed of the order parameter components $\eta_{i}$ and the components of the strain tensor $\varepsilon_{\mathrm{ij}}: \mathrm{F}_{\mathrm{c}}=\sum_{\mathrm{l}} \mathrm{J}_{\mathrm{l}}\left(\eta_{\mathrm{i}}, \varepsilon_{\mathrm{ij}}\right)$. They are invariant with respect to the direct sum of the representations $\tau=\tau(\eta) \oplus \tau(\varepsilon)$ of the parent phase space group $G$, where $\tau(\eta)$ is the irreducible representation associated with the order parameter and $\tau(\varepsilon)$ - with the strain tensor.

In summary, phase transition in the $3 \mathrm{D}$ metal-oxide interface can be considered as the order-disorder phase transition in the oxygen lattice of the parent phase submitted to the long-range anisotropic diffusional field induced by the metal substrate and to the isotropic elastic field due to the oxygen-oxygen interaction. The Landau-Ginzburg free energy of the system can be written as :

$$
\overline{\mathrm{F}}=\int_{\mathrm{V}}\left\{\mathrm{F}\left(\eta_{\mathrm{i}}\right)+\frac{1}{2} \mathrm{~g}\left(\nabla \eta_{\mathrm{i}}\right)^{2}+\mathrm{F}_{\mathrm{el}}\left(\varepsilon_{\mathrm{ij}}\right)+F_{\mathrm{c}}\left(\eta_{\mathrm{i}}, \varepsilon_{\mathrm{ij}}\right)\right\} \mathrm{dV}-\int_{\mathrm{S}} \mathrm{P}_{\mathrm{i}} \mathrm{u}_{\mathrm{i}} \mathrm{dS}
$$

where the first and the second terms represent the free energy of the ordering taken in the form of the Landau expansion, third one is the elastic energy of the cubic solid, $V$ is the solid volume, $S$ is that part of the surface to which the force $P_{i}$ is applied, $u_{i}$ is the displacement vector. If the characteristic length of inhomogeneities in the interface $L>>\zeta$, where $\zeta$ is the OP correlation length, the gradient term in Eq. (1) can be neglected. Then, in absence of the external force, the free energy of the considered system takes the form : $F=F\left(\eta_{i}\right)+F_{e l}\left(\varepsilon_{i j}\right)+F_{c}\left(\eta_{i}, \varepsilon_{i j}\right)$. The equilibrium state of the system is defined by the minimization of the free energy with respect to the order parameter components $\eta_{i}$ and the components of the strain tensor $\varepsilon_{\mathrm{ij}}$. (Note that in general case one should use more sophisticated conditions of the mechanical equilibrium, see f.e. $[19,49-52]$.)

\subsection{Oxidation of the (001) surface of iron}

Let us now illustrate the preceding ideas and the results of the proposed model on the example of the oxidation of the (001) Fe surface in its monooxide. For that score let us precise the groups of symmetry and lattice parameters of the both constituants, together with their orientation relationship [53]: Fe : $\mathrm{O}_{\mathrm{h}}^{9}$ space group $(\mathrm{Fm} \overline{3} \mathrm{~m})$, b.c.c. lattice, lattice parameter $\mathrm{a}=2.86 \mathrm{~A} ; \mathrm{FeO}: \mathrm{O}_{\mathrm{h}}^{5}$ space group $(\operatorname{Im} \overline{3} \mathrm{~m})$, f.c.c. lattice, $\mathrm{NaCl}$-type structure, lattice parameter $\mathrm{a}^{\prime}=4.28 \mathrm{~A}$ The orientation relationship between the $\mathrm{Fe}$ lattice and $\mathrm{FeO}$ lattice in this case can be summarized as : $(001) \mathrm{Fe} / /(001) \mathrm{FeO} ;[001] \mathrm{Fe} / /[001] \mathrm{FeO}$; [110]Fe // [100]FeO. Maximal common subgroup of the point groups of Fe and FeO for this orientation is evidently $\mathrm{D}_{4 \mathrm{~h}}(4 / \mathrm{mmm})$. The basic translations of the $3 \mathrm{D}$-interface : $\overline{\mathrm{T}}_{1}, \overrightarrow{\mathrm{T}}_{2}$ and $\overline{\mathrm{T}}_{3}$ satisfying orientation relationship and lattice parameters ratio, can be defined as following combinations of the basic translations of $\mathrm{Fe}$ or basic translations of $\mathrm{FeO}$ (up to the symmetry-nonbreaking deformations) : $\overline{\mathrm{T}}_{1} \approx \overline{\mathrm{a}}_{1}^{\mathrm{FeO}} \approx \overrightarrow{\mathrm{a}}_{1}^{\mathrm{Fe}}+\overrightarrow{\mathrm{a}}_{2}^{\mathrm{Fe}}$ and $\overrightarrow{\mathrm{T}}_{2} \approx \mathrm{a}_{2}^{\mathrm{FeO}} \approx-\overline{\mathrm{a}}_{1}^{\mathrm{Fe}}+\overline{\mathrm{a}}_{2}^{\mathrm{Fe}}$ because of the relation $(001) \mathrm{Fe} / /(001) \mathrm{FeO}$ and [110]Fe // [100] FeO ; $\overline{\mathrm{T}}_{3} \approx 2 \overrightarrow{\mathrm{a}}_{3}^{\mathrm{FeO}} \approx 3 \overline{\mathrm{a}}_{3}^{\mathrm{Fe}}$ due to the $\mathrm{a}^{1 / \mathrm{a}} \approx 3 / 2$ and $[001] \mathrm{Fe} / /[001] \mathrm{FeO}$, where $\overrightarrow{\mathrm{a}}_{1}^{\mathrm{FeO}}, \overrightarrow{\mathrm{a}}_{2}^{\mathrm{FeO}}$, are the basic translations of the oxide lattice and $\mathrm{a}_{1}^{\mathrm{Fe}}, \overrightarrow{\mathrm{a}}_{2}^{\mathrm{Fe}}, \overrightarrow{\mathrm{a}}_{3}^{\mathrm{Fe}}$ are the basic translations of the metal lattice.

In the case of the dominant cation diffusion mode one can neglect exact group-subgroup relationship between the space groups of the metal and of the 3D-interface. By contrast, the interface must satisfy such kind of relation with respect to the parent phase, presented here by the f.c.c. lattice of oxygen : $\overrightarrow{\mathrm{T}}_{1}=\overrightarrow{\mathrm{a}}_{1}^{\mathrm{O}}, \overline{\mathrm{T}}_{2}=\overline{\mathrm{a}}_{2}^{\mathrm{O}}$ and $\overrightarrow{\mathrm{T}}_{3}=2 \overrightarrow{\mathrm{a}}_{3}^{\mathrm{O}}$. Taking into account all these facts it is easy to obtain the space group and the crystallographic structure (up to the deformations) of the $3 \mathrm{D}$-interface. Resulting space group is $\mathrm{D}_{4 \mathrm{~h}}^{1}$ ( $\mathrm{P} 4 / \mathrm{mmm}$ ), corresponding structure is presented in Fig. 1. It is four-layered with different probabilities of occupation of the layers by $\mathrm{Fe}$ atoms.

Introduction of the 3D-structure of the interface permits to estimate the limits of the growth deformation and the "effective" Pilling-Bedworth Ratio by comparing the lattice parameters of the intermediate tetragonal phase in the vicinity of the metal and in the vicinity of the equilibrium oxide. Indeed, in the vicinity of the oxide the relation between the parameters is : 
in the vicinity of the metal:

$$
\left|\overrightarrow{\mathrm{T}}_{1}^{\mathrm{f}}\right|=\left|\overrightarrow{\mathrm{a}}_{1}^{\mathrm{FeO}}\right|,\left|\overrightarrow{\mathrm{T}}_{2}^{\mathrm{f}}\right|=\left|\overrightarrow{\mathrm{a}}_{2}^{\mathrm{FeO}}\right| \text { and }\left|\overrightarrow{\mathrm{T}}_{3}^{\mathrm{f}}\right|=2\left|\overrightarrow{\mathrm{a}}_{3}^{\mathrm{FeO}}\right|
$$

$$
\left|\overrightarrow{\mathrm{T}}_{1}^{\mathrm{I}}\right|=\left|\overrightarrow{\mathrm{a}}_{1}^{\mathrm{Fe}}+\overrightarrow{\mathrm{a}}_{2}^{\mathrm{Fe}}\right|,\left|\overrightarrow{\mathrm{T}}_{2}^{\mathrm{I}}\right|=\left|-\overrightarrow{\mathrm{a}}_{1}^{\mathrm{Fe}}+\overrightarrow{\mathrm{a}}_{2}^{\mathrm{Fe}}\right|,\left|\overrightarrow{\mathrm{T}}_{3}^{\mathrm{I}}\right|
$$

and $\left|\vec{T}_{3}^{I}\right|$ can vary from $\left|\vec{T}_{3}^{\prime}\right|=2 \sqrt{2}\left|\vec{a}_{3} \mathrm{Fe}^{\prime}\right|$ in the ideal case, when the intermediate phase is composed of two nondeformed cells of the f.c.c. lattice of oxygen, to $\left|\overrightarrow{\mathrm{T}}_{3}^{\prime \prime}\right|=3\left|\overrightarrow{\mathrm{a}}_{3}^{\mathrm{Fe}}\right|$ in the case of strong ordering of Fe atoms, which deforms the oxygen lattice from the very beginning of the oxidation process. Here $\vec{T}_{m}^{I}$ and $\vec{T}_{m}^{f}(m=1,2,3)$ are initial and final tetragonal translations. The deformation associated with the difference of $\overrightarrow{\mathrm{T}}_{m}^{\mathrm{I}}-\overrightarrow{\mathrm{T}}_{\mathrm{m}}^{\mathrm{f}}$ has the value :

$$
\varepsilon_{x x}^{\max }=\varepsilon_{y y}^{\max }=\frac{a^{\prime}-a \sqrt{2}}{a \sqrt{2}}=5.8 \% ; \varepsilon_{z z}^{\max }=\frac{2 a^{\prime}-2 \sqrt{2} a}{2 \sqrt{2} a}=5.8 \% ; \varepsilon_{z z}^{\min }=\frac{2 a^{\prime}-3 a}{3 a}=-0.23 \%
$$

Analogousiy, one can define "effective" PBR, calculated in the fixed crystallographic system of axes, as $(\mathrm{PBR})_{\text {eff }}=\left(\mathrm{V}_{\mathrm{f}} / \mathrm{V}_{\mathrm{I}}\right)$, which can vary from its minimal value $(\mathrm{PBR})_{\mathrm{eff}}^{\mathrm{min}}$ :

$\left(a^{3}\left|\begin{array}{lll}1 & 0 & 0 \\ 0 & 1 & 0 \\ 0 & 0 & 2\end{array}\right|\right) /\left(a^{3}\left|\begin{array}{ccc}1 & 1 & 0 \\ -1 & 1 & 0 \\ 0 & 0 & 3\end{array}\right|\right)=\frac{2 a^{3}}{6 a^{3}}=1,12$ to $(P B R)_{\mathrm{eff}}^{\max }=1,18$ in the case when $\left|\vec{T}_{3}^{\prime}\right|=2 \sqrt{2} \mid \vec{a}=$ Fe $\mid$ in the ideal disordered and non-deformed initial state of oxidation. These values differ greatly from the classical PBR value for $\mathrm{Fe} / \mathrm{FeO}$ system, which is equal to $1.68[8,26]$, but it is interesting to note that the value of the ratio (PBR) eff $f$ PBR is very close to the empirical factor introduced in the calculations of the stresses due to the PBR to make these stresses more reliable $[54,55]$.

a)

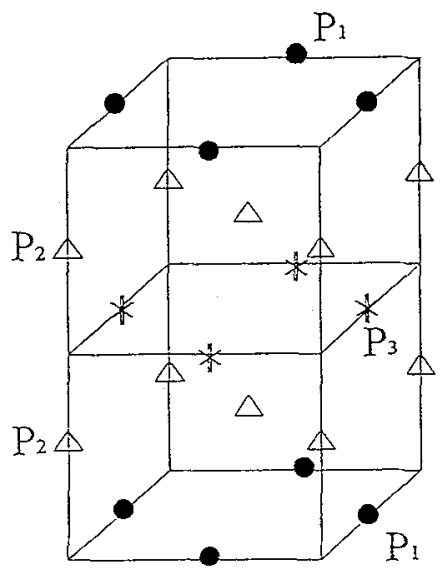

b)

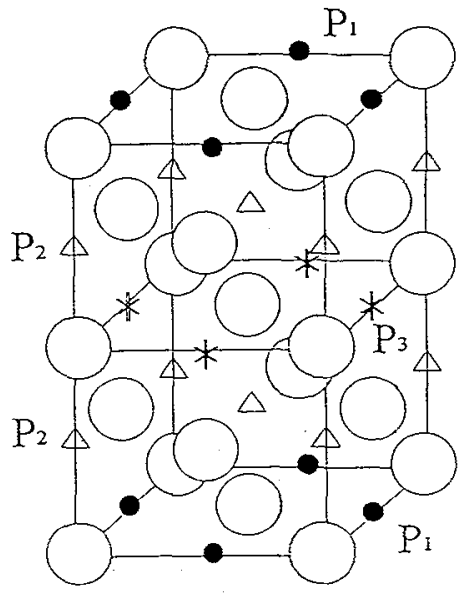

Figure 1: Crystallographic tetragonal structure of the ordered $\mathrm{Fe} / \mathrm{FeO}$ interface. a) metallic sublattice with the atomic positions occupied with different probability ; b) complete structure

Crystallographic structure of the interface allows also to find the order parameter $(O P)$ of the order-disorder phase transition. It is associated with the wave vector $\vec{k}=\vec{k}_{6}=\tau\left(\begin{array}{lll}0 & 0 & \frac{1}{2}\end{array}\right)=\frac{1}{4}\left(\begin{array}{ll}\vec{b}_{1}+\vec{b}_{2}\end{array}\right)$. The notations of this vector are respectively, i) Kovalev classification [56] ; ii) expression in the Cartesian reciprocal space ; iii) expression in terms of the basic vectors of the reciprocal space of the f.c.c. lattice $\bar{b}_{\mathrm{k}}$. The order parameter has six components: $\left\langle\eta_{1} \quad \eta_{2} \quad \eta_{3} \quad \eta_{4} \quad \eta_{5} \quad \eta_{6}\right\rangle$. Introducing complex notations $C_{1}=\eta_{1}+\eta_{2} ; C_{2}=C_{1} * C_{3}=$ $\eta_{3}+i_{4} ; C_{4}=C_{3} *$ etc. one can express $C_{i}$ as a function of the probabilities of occupation of the interstices in the different layers of the oxygen lattice as: 


$$
C_{i}=\frac{1}{\left\|d_{i}\right\|} \sum_{j} e^{2 \pi i \vec{k}_{i} \cdot \vec{r}_{j}} P_{j}
$$

where $\overrightarrow{\mathrm{k}}_{\mathrm{i}}$ runs over six arms of the star of the wave vector and $\overrightarrow{\mathrm{r}}_{\mathrm{j}}$ runs over four layers of the structure (Fig. 1). For example, $\mathrm{C}_{1}=1 / 2\left(\mathrm{P}_{1}+\mathrm{i} \mathrm{P}_{2}-\mathrm{P}_{3}-\mathrm{i} \mathrm{P}_{4}\right)$, etc. The metal concentration $\mathrm{x}_{\mathrm{m}}$ in the same notations can be written as $\mathrm{x}_{\mathrm{m}}=1 / 4\left(\mathrm{P}_{1}+\mathrm{P}_{2}+\mathrm{P}_{3}+\mathrm{P}_{4}\right)$.

The free energy (1) of the stress-free non-deformed oxygen lattice have to be invariant with respect to the direct sum of the representation of the $O P$ and of the strain tensor representation. In the following consideration we will limit the free energy by the fourth-degree expansion. Then, the term $F\left(\eta_{i}\right)$ will contain following invariants :

$$
\begin{aligned}
& \mathrm{I}_{1}=\eta_{1}^{2}+\eta_{2}^{2}+\eta_{3}^{2}+\eta_{4}^{2}+\eta_{5}^{2}+\eta_{6}^{2} \\
& \mathrm{I}_{2}=\eta_{1}^{4}-6 \eta_{1}^{2} \eta_{2}^{2}+\eta_{2}^{4}+\eta_{3}^{4}-6 \eta_{3}^{2} \eta_{4}^{2}+\eta_{4}^{4}+\eta_{5}^{4}-6 \eta_{5}^{2} \eta_{6}^{2}+\eta_{6}^{4} \\
& \mathrm{I}_{3}=\left(\eta_{1}^{2}+\eta_{2}^{2}\right)^{2}+\left(\eta_{3}^{2}+\eta_{4}^{2}\right)^{2}+\left(\eta_{5}^{2}+\eta_{6}^{2}\right)^{2},
\end{aligned}
$$

elastic free energy is $\mathrm{F}_{\mathrm{el}}=\frac{1}{2} \lambda \varepsilon_{\mathrm{I}}^{2}+2 \mu \varepsilon_{\mathrm{II}}$, with $\varepsilon_{\mathrm{I}}=\operatorname{Tr}(\varepsilon)=\varepsilon_{\mathrm{ii}}$ and $\varepsilon_{\mathrm{II}}=\frac{1}{2} \operatorname{Tr}\left(\varepsilon^{2}\right)=\frac{1}{2} \varepsilon_{\mathrm{ij}} \varepsilon_{\mathrm{ij}}$ and the coupling term $\mathrm{F}_{\mathrm{c}}$ contains two invariants :

$$
\begin{aligned}
& \mathrm{J}_{1}=\left(\eta_{1}^{2}+\eta_{2}^{2}\right) \varepsilon_{\mathrm{zz}}+\left(\eta_{5}^{2}+\eta_{6}^{2}\right) \varepsilon_{\mathrm{xx}}+\left(\eta_{3}^{2}+\eta_{4}^{2}\right) \varepsilon_{\mathrm{yy}} \\
& \mathrm{J}_{2}=\left(\eta_{1}^{2}+\eta_{2}^{2}\right) \varepsilon_{\mathrm{xx}} \varepsilon_{\mathrm{yy}}+\left(\eta_{5}^{2}+\eta_{6}^{2}\right) \varepsilon_{\mathrm{yy}} \varepsilon_{\mathrm{zz}}+\left(\eta_{3}^{2}+\eta_{4}^{2}\right) \varepsilon_{\mathrm{xx}} \varepsilon_{\mathrm{zz}}
\end{aligned}
$$

The $\mathrm{D}_{4 \mathrm{~h}}^{1}(\mathrm{P} 4 / \mathrm{mmm})$ structure of the $3 \mathrm{D}$-interface corresponds to the following relations between the components of the OP : $C_{1}=C_{1}{ }^{*}, C_{2}=C_{3}=0\left(\begin{array}{llllll}(C & C & 0 & 0 & 0 & 0\end{array}\right)$ or $\eta_{1}=0, \eta_{i}=0(i=2-6)$. This implies $\mathrm{P}_{2}=\mathrm{P}_{4}$ in all phases, consequently the phase transition is described completely by only one component of the OP $\eta=\eta_{1}=1 / 2\left(P_{1}-P_{3}\right)$. Using this fact we can simplify the free energy of the transition, which takes the form:

$$
\mathrm{F}=\frac{1}{2} \mathrm{a}_{1} \eta^{2}+\frac{1}{4} \eta^{4}+\frac{\gamma}{2} \eta^{2} \varepsilon_{\mathrm{zz}}+\frac{\delta}{2} \eta^{2} \varepsilon_{\mathrm{xx}} \varepsilon_{\mathrm{yy}}+\frac{1}{2} \lambda\left(\varepsilon_{\mathrm{xx}}+\varepsilon_{\mathrm{yy}}+\varepsilon_{\mathrm{zz}}\right)^{2}+\mu\left(\varepsilon_{\mathrm{xx}}^{2}+\varepsilon_{\mathrm{yy}}^{2}+\varepsilon_{\mathrm{zz}}^{2}\right)
$$

here $\mathrm{a}_{1}=\alpha\left(\mathrm{T}-\mathrm{T}_{\mathrm{c}}\right), \gamma$ and $\delta$ are the phenomenological constants expressing the strength of the coupling between thermodynamic and elastic subsystems. The equilibrium state of the interface is obtained by the minimization of the free energy (2) with respect to $\eta$ and by the conditions of the elastic equilibrium:

$$
\frac{\partial \mathrm{F}}{\partial \eta}=0 ; \quad \frac{\partial \mathrm{F}}{\partial \varepsilon_{\mathrm{xx}}}=\sigma_{\mathrm{xx}} ; \quad \frac{\partial \mathrm{F}}{\partial \varepsilon_{\mathrm{yy}}}=\sigma_{\mathrm{yy}} ; \quad \frac{\partial \mathrm{F}}{\partial \varepsilon_{\mathrm{zz}}}=\sigma_{\mathrm{zz}}=0
$$

The component of the internal stress $\sigma_{z z}$ is equal to zero because of the boundary conditions : in this direction the scale is not fixed. The equations of state (3) describe two phases of different symmetry, the first one $\eta=0$, which corresponds to the cubic oxygen lattice and/or to the equilibrium oxide. The difference consists only in the $\mathrm{Fe}$ ions concentration. The second phase, $\eta=0$ corresponds to the tetragonal $3 D$-interface. In both phases one can use the notations $\varepsilon=\varepsilon_{x x}=\varepsilon_{y y} ; \sigma=\sigma_{x x}=\sigma_{y y}$.

The resulting phase diagram of the system is shown in the Fig. 2. In the first approximation it can be considered as the temperature-thickness phase diagram. i) For the high temperatures $T>T_{m}$, where $T_{m}$ is defined by the condition $a_{1 m}=\alpha\left(T_{m}-T_{c}\right)=\frac{\gamma^{2} \lambda^{2}}{\delta(\lambda+2 \mu)}$, the phase $\eta=0$ is stable for all values of $\sigma$ and, consequently for all values of the scale thickness. For these temperatures thermal disorder is dominant and it destroys the ordering of $\mathrm{Fe}$ ions. The diffusion is isotropic and the interface remains always cubic (path 1 in Fig. 2). ii) For the temperatures $T<T_{m}$ the region of stability of the tetragonal phase appears on the phase diagram. It is limited by the curve : $a_{1}-\lambda \gamma \bar{\sigma}+\delta\left(\frac{\lambda+2 \mu}{2}\right)^{2} \bar{\sigma}^{2}=0$, where $\bar{\sigma}=\frac{\sigma}{\mu(3 \lambda+2 \mu)}$. The critical value of $\sigma$, for which this region of stability appears is $\sigma_{\mathrm{m}}=\frac{2 \gamma \lambda \mu(3 \lambda+2 \mu)}{\delta(\lambda+2 \mu)}$. Along the path 2 in Fig. 2 the initially cubic interface undergoes second order phase transition into the tetragonal state and it 
becomes again cubic for the bigger values of $\sigma$ (and of the scale thickness). iii) For the temperatures $\mathrm{T}<\mathrm{T}_{\mathrm{trc}}$, where $\mathrm{T}_{\mathrm{trc}}$ is defined by the condition $\mathrm{a}_{\mathrm{trc}}=\alpha\left(\mathrm{T}_{\mathrm{trc}}-\mathrm{T}_{\mathrm{c}}\right)=\frac{\gamma^{2}(\lambda+\mu)-2 \mu(3 \lambda+2 \mu)}{\delta(\lambda+2 \mu)}$, the order of the phase transition changes from second to first (path 3 in Fig. 2). There is no more possibility of continuous evolution of the interface, neither in the vicinity of the metal nor near the oxide. Thus, the phases become structurally incompatible. It means that in the region of the coexistence of the two phases the interface must loose its continuity. This is a necessary condition of grains formation, it is related also to the fracture and other types of the scale failure. iv) For the temperatures $\mathrm{T}<\mathrm{T}_{\mathrm{c}}$, the $3 \mathrm{D}$-interface grows as the tetragonal ordered phase since the first scale layer (path 5 in Fig. 2) at it is again compatible with the substrate. By contrast, the structural incompatibility with the equilibrium oxide becomes more pronounced.

The dependencies of the in-layer deformation $\varepsilon$ on the value of $\sigma$ corresponding to the different paths on the phase diagram are given in Fig. 3. These paths can be associated with the different isothermal growth processes of the $\mathrm{FeO}$ scale. In the cubic phase there is only elastic contribution to the strain and $\varepsilon$ is linearly related to $\sigma: \varepsilon=\sigma \frac{\lambda+2 \mu}{2 \mu(3 \lambda+2 \mu)}$ and $\varepsilon_{Z Z}=-\sigma \frac{\lambda}{\mu(3 \lambda+2 \mu)}$. In the tetragonal phase the OP contributes to the deformation and the relation between $\varepsilon$ and $\sigma$ is described by the equation :

$$
\mathrm{p}^{3}+\mathrm{p}\left(\mathrm{b} \delta(\lambda+2 \mu)^{2}+2 \mu(3 \lambda+2 \mu) \Delta\right)-\delta \mathrm{r}(\lambda+2 \mu)^{2} \Delta=0
$$

where $\mathrm{p}=\varepsilon \delta(\lambda+2 \mu)-\gamma \lambda, \mathrm{b}=\mathrm{a}_{1}-\mathrm{a}_{1 \mathrm{~m}}, \mathrm{r}=\sigma-\sigma_{\mathrm{m}}$ and $\Delta=\gamma^{2}-2(\lambda+2 \mu)$.

The dependencies of the OP value on $\sigma$ are shown in Fig. 4.

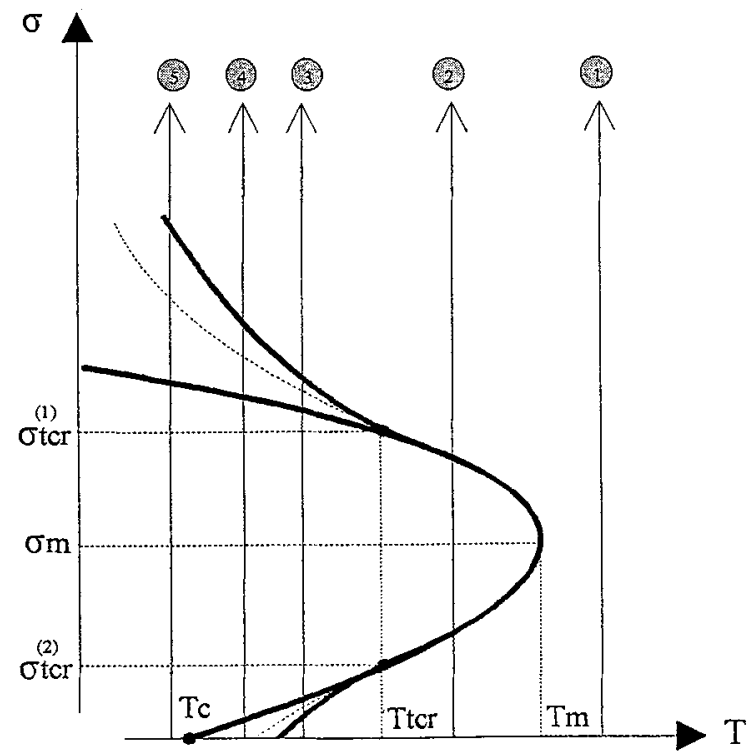

Figure 2: Phase diagram of the model of the Fe/FeO interface, described by the free energy (2). (Solid lines represent stability boundaries. Ttrc indicates tricritical point, in which the order of the transition changes) Different paths correspond to the different isothermal growth processes.

\section{DISCUSSION}

The present work can be taken as the first approximation of the growth processes in the 3D-interface of the metal-oxide undergoing phase transition. It takes into account mainly the symmetry of the problem and relates it to the invariant properties of the diffusion mode and of the elastic strain. Such approach should give satisfactory qualitative picture of different isothermal growth regimes. Resulting $\varepsilon-\sigma$ dependencies (Fig. 3) for different paths in the phase diagram (Fig. 2) can be useful in the interpretation of the strains measured by means of the X-ray diffraction and calculation of the stresses on their basis. The results 
obtained by the structure analysis can be already used in the fine modelization of the stresses, similar to the procedure given in [55].

There are many ways of generalization of the proposed method. One of them is to consider the general case of the 3D-interface incommensurate with respect both to the substrate and to the oxide structure, with the possibility of the wave vector dependence on temperature and thickness. Another one is to study the interfaces of the thick scales. In this case a simple free energy expansion should be replaced by the differential equations of diffusion for each type of interstices and for the macroscopic average concentration. To obtain the stresses induced in the system it is necessary to solve these equations together with the differential equations of the mechanical equilibrium.
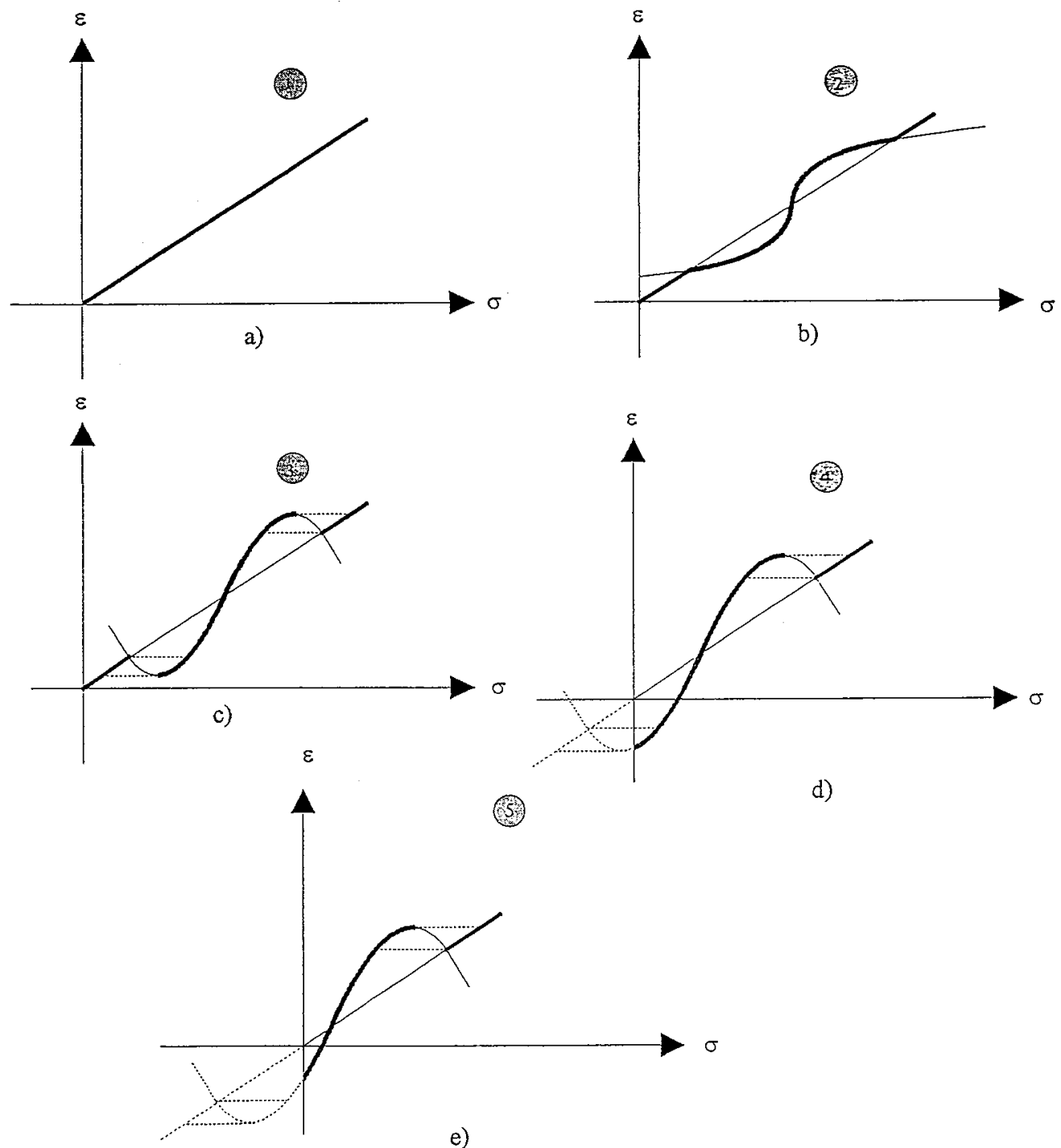

d)

e)

Figure 3: Dependence of the in-plane deformation $\varepsilon$ of the interface on the internal stress $\sigma$ applied to the parent phase lattice ( $\sigma$ increases with the scale thickness) along the different paths on the phase diagram (Fig. 2). 


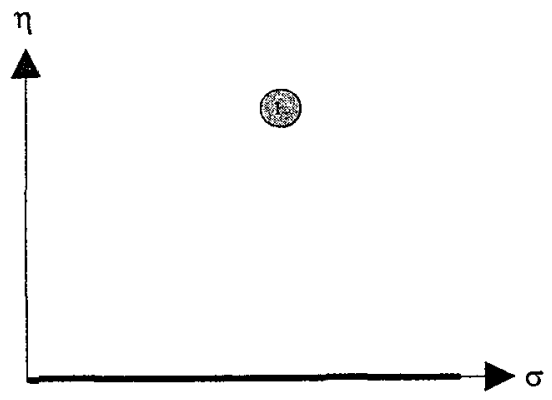

a)

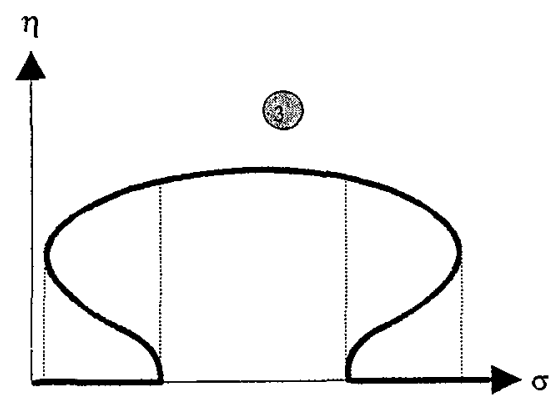

c)

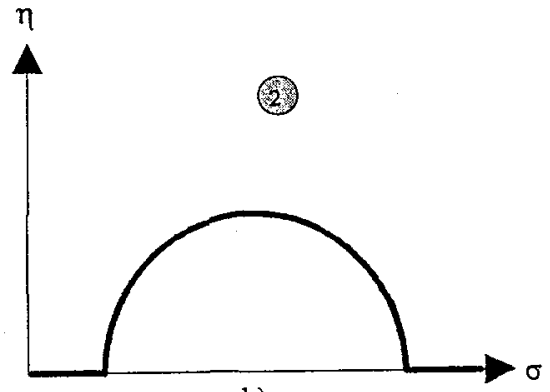

b)

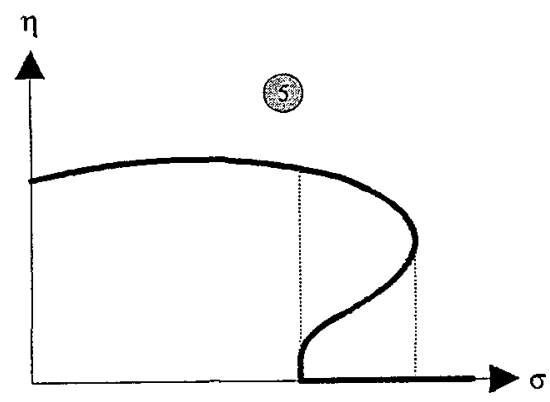

e)

Figure 4: Dependence of the order parameter $\eta$ of the order-disorder transformation on the internal stress $\sigma$ applied to the parent phase lattice ( $\sigma$ increases with the scale thickness) along the different paths on the phase diagram (Fig. 2).

\section{Acknowledgements}

The authors acknowledge Région de Picardie for its financial support for this study.

\section{References}

[1] Stringer J., Corros. Sci. 10 (1970) 513

[2] Rhines R.N. and Wolf J.S., Metall. Trans. 1 (1970) 1701-1710

[3] Stringer J., J. Less-Common Metals 16 (1968) 55-64

[4] Roy C. and Burgess B., Oxid. of Metals 2 (1970) 235-261

[5] Francis J.M. and Hodgson K.E., Mat. Sci. Eng. 6 (1970) 313-319

[6] Douglas D.L., Oxid. of Metals 1 (1969) 127

[7] Cathcart J.V., in High-Temperature Gas-Metal Reactions in Mixed Environments (A IME, New York, 1973) p. 63

[8] Fontana M.G. and Staehle R.W. (Eds.), Advances in Corrosion Science and Technology, Vol. 4 (Plenum Press, New York, 1974)

[9] Cathcart J.V. (Ed.), Stress Effect and the Oxidation of Metals (AIME, New York, 1975)

[10] Beranger G. and Coddet, J. Microsc. Spectrosc. Electron. 5 (1980) 793

[11] Beranger G., Colson J.S. and Dabosi F. (Eds.) Corrosion des Matériaux à Haute Température (Les Editions de Physique, Paris, 1985)

[12] Noyan J.C. and Cohen J.B., Residual Stress: Measurement by Diffraction and Interpretation (Spinger, New York, 1987)

[13] Huntz A.M., Mater Sci. Technol. 4 (1988) 1079

[14] Aubry A., Armanet F., Beranger G., Lebrun G.L., G. Maeder, Acta Metall. 36 (1988) 2779-2786 
[15] Härting M. and Fritsch G., J. Phys. D : Appl. Phys. 26 (1993) 1814-1816

[16] Liu C., Huntz A.M., Lebrun J.L., Mat. Sci. Eng. A160 (1993) 113-126

[17] Huntz A.M., Liu C., Kornmeier M., Lebrun J.L., Corros. Sci. 35 (1993) 989-997

[18] Ruud J.A., Witvrouw A., and Spaepen F., J. Appl. Phys. 74 (1993) 2517-2523

[19] Bulbich A.A., J. Mat. Sci. 27 (1992) 6401-6410

[20] Gufan Yu.M., Kutyin E.I., Lorman V.L., Sidorenko E.N., Sov. Phys. : Solid State, 29 (1987) 756

[21] Baxter D.J. and Natesan K., Rev. of High-Temp. Mat. 3-4 (1983) 149-249

[22] Borie B.S., Sparks C.S., and Cathcart J.V., Acta. Met. 10 (1962) 691

[23] Norin A., Oxid. Met., 9 (1975) 259

[24] Gwathmey A.T. and Young F.W., Rev. Met. 48 (1951) 434

[25] Landau L.D. and Lifshitz E.M., Statistical Physics (Pergamon Press, London, 1969)

[26] Holmes D.R. and Pascoe R.T., Wekstoffe und Korrosion 23 (1972) 859

[27] Gleiter H., Mat. Sci. Eng. 52 (1982) 91-31

[28] Chadwick G.A. and Smith D.A. (Eds.), Grain Boundary Structure and Properties (Academic Press, New York, 1976)

[29] Jonson W.C. and Blakely J.M. (Eds.), Interfacial Segregation (American Society for Metals, Metals Park, OH, 1979)

[30] Kronberg M.L. and Wilson F.H., Trans. AME 185 (1949) 501

[31] Arkharov V.I., Fiz. Met. Metalloved. 12 (1961) 223

[32] Ranganathan S., Acta Crystallogr. 21 (1966) 197

[33] Bollmann W., Philos. Mag. 16 (1967) 363 and Bollmann W., Philos. Mag. 16 (1967) 383

[34] Bollmann W., Crystal Defects and Crystalline Interfaces (Springer, Berlin, 1970)

[35] Warrington D.H. and Bollmann W., Philos. Mag. 25 (1972) 1195

[36] Simpson C.J., Aust K.T. and Winegard W.C., Metall. Trans. 2 (1971) 987

[37] Demianczuk D.W. and Aust K.T., Acta Metall. 23 (1975) 1140

[38] Gleiter H., Radex Rundsch., 1 (1980) 51

[39] Kikuchi R. and Cahn J.W., Phys. Rev. B, 21 (1980) 1893

[40] Erb U. and Gleiter H., Scr. Metall., 13 (1979) 61

[41] Meisser H., Gleiter H. and Mirwald E., Scr. Metall., 14 (1980) 95

[42] Landau L.D., Collected papers of L.D. Landau (Ed. D; Ter Haar, Pergamon, London, 1965) p. 193

[43] Gufan Yu. M., Structurnye Fazovye Perehody (Structural Phase Transitions, Nauka, Moscow, 1982)

[44] Khachaturyan A.G., Theory of Structural Transformations in Solids (John Wiley \& Sons, New York, 1983)

[45] Toledano J.C. and Toledano P., The Landau Theory of Phase Transitions (World-Scientific,

Singapore, 1987)

[46] Dmitriev V.P. et al, Phys. Rev. Lett., 60 (1988) 1958-1961

[47] Dmitriev V.P. et al, Phys. Rev. Lett., 62 (1989) 2495-2498

[48] Dmitriev V.P., Rochal S.B., Lorman V.L. and Toledano P., Phys. Rev. B, 49 (1994) 838-843

[49] Landau L.D. and Lifshitz E.M., Elasticity theory (Pergamon, London, 1969)

[50] Bulbich A.A., J. Mat. Sci., 27 (1992) 1070

[51] Bulbich A.A. and Gufan Yu.M., Ferroelect. 98 (1989) 589

[52] Bulbich A.A. and Pumpyan P.E., Ferroelect. 111 (1990)

[53] Bardolle J., Orientation des produits de réaction à la surface d'un metal, in Oxydation des Metaux (Gautier-Villars, Paris)

[54] Bernstein H.L., Metall. Trans. 18A (1987) 975-986

[55] Touati A., Roelandt J.M., Armanet F. and Beranger G., "Simulation of the behaviour of high temperature oxidized structures (application to coating)", International Conference on Advanced

Materials- ICAM 91, Strasbourg, 1991(Elsevier Science Publishers, North Holland, 1992) p. 233

[56] Kovalev O.V., Irreducible Representations of the Space Groups (Gordon and Breach, New York, 1965) 